\title{
Carregamentos Dinâmicos Induzidos Pelos Movimentos de Pedestres em Estruturas
}

\section{Dynamic Loads Induced by Pedestrian Movements in Structures}

\author{
Pedro Gutemberg de Alcântara Segundinho' ${ }^{1}$; Antonio Alves Dias²; \\ Marcelo Rodrigo Carreira ${ }^{3}$
}

\begin{abstract}
Resumo
Os engenheiros civis há algum tempo estão cientes dos problemas causados pelas vibrações excessivas provocadas pelo fenômeno da ressonância nas passarelas. As fontes causadoras desse fenômeno são várias e tem origens diferentes como, por exemplo, pedestres praticando alguns modos de caminhada, aglomerações excepcionais de pedestres, ventos fortes, chuva etc. $\mathrm{O}$ objetivo deste trabalho é apresentar uma revisão bibliográfica que possibilite o entendimento dos tipos de carregamentos dinâmicos que são introduzidos nas estruturas devido aos movimentos de pedestres. O estudo foi desenvolvido a partir da análise teórica de diversos autores a respeito do movimento de pedestres em estruturas. As várias propostas de modelagem permitem utilizar até cinco harmônicos da ação dos pedestres ou apenas aquele que considera o maior efeito de ressonância. Constatou-se que as modelagens propostas se diferenciam, principalmente, pelo número de harmônicos considerados durante a ação dos pedestres.

Palavras-chave: Movimento de pedestres. Frequências de caminhada. Vibrações em estruturas. Conforto humano.
\end{abstract}

\begin{abstract}
Civil engineers for being sometimes aware of the problems caused by excessive vibration caused by the resonance phenomenon on the footbridges. The sources that cause this phenomenon are several and have different origins, for example, pedestrians practicing some ways of walking, exceptional agglomerations of pedestrians, strong winds, rain etc. The objective of this paper is to present a literature review that allows understanding of the types of dynamic loads that are introduced in the structures due to pedestrian movements. The study was developed from the theoretical analysis of several authors about the movement of pedestrians in structures. The various modeling proposals allow the use of up to five pedestrian action harmonics or only those that consider the greatest resonance effect. It was verified that the proposed models are differentiated, mainly, by the number of harmonics considered during the action of pedestrians.
\end{abstract}

Keywords: Pedestrians movements. Walking frequencies. Vibrations in structures. Human comfort.

\footnotetext{
${ }^{1}$ Professor Doutor, Departamento de Ciências Florestais e Madeira, Centro de Ciências Agrárias e Engenharias, Universidade Federal de Espirito Santo, Av. Gov. Lindemberg, 316, 29550-000, Jerônimo Monteiro, ES, Brasil. E-mail: p_gutemberg2001@yahoo.com.br

${ }^{2}$ Professor Doutor, Departamento de Engenharia de Estruturas, Escola de Engenharia de São Carlos, Universidade de São Paulo, Av. Trabalhador São-carlense, 400, 13566-590, São Carlos, SP, Brasil. E-mail: dias@ @sc.usp.br

${ }^{3}$ Professor Doutor, Coordenação de Engenharia Civil, Campus Campo Mourão, Universidade Tecnológica Federal do Paraná, Rodovia BR 369, km 0,5, 87301-006, Campo Mourão, PR, Brasil. E-mail: carreira@utfpr.edu.br
} 


\section{Introdução}

Os engenheiros civis há algum tempo estão cientes dos problemas causados pelas vibrações excessivas provocadas pelo fenômeno da ressonância nas passarelas. As fontes causadoras desse fenômeno são várias, podendo ter aspetos diferentes como, por exemplo, pedestres praticando alguns modos de caminhada, aglomerações excepcionais de pedestres, ventos fortes, chuva, passagem de veículos pesados sob elas etc. $\mathrm{O}$ fenômeno da ressonância ocorre devido à proximidade entre as frequências de excitação relativas às fontes e as frequências naturais da estrutura, que aumenta as amplitudes de deslocamentos provocando desconforto. Além desse aspecto, é importante frisar que a amplitude da vibração também é influenciada pelo amortecimento da estrutura.

Em algumas situações, a atividade de caminhar, quando realizada sobre passarelas, produz vibrações excessivas que são desconfortáveis para os seres humanos e prejudiciais para essas estruturas. É importante ressaltar que o estudo do fenômeno das vibrações excessivas em passarelas causadas por pedestres não é recente no mundo. Mas, nas últimas décadas tem ocorrido uma demanda maior desse tipo de estrutura em virtude do grande aumento da população, tanto urbana quanto rural, e assim os problemas de vibrações excessivas em passarelas têm sido mais evidentes. Também ocorreu melhora na qualidade dos materiais utilizados e, como consequência, melhora nas propriedades mecânicas dos materiais usados na construção dessas estruturas que, privilegiando a estética, tendem a ser cada vez mais leves e esbeltas. Começou-se então a perceber uma maior susceptibilidade a vibrações excessivas dessas estruturas quando submetidas a carregamento dinâmico induzido por pedestres. As demais fontes causadoras de vibrações excessivas nas passarelas não foram levadas em conta neste trabalho, devido à sua menor ocorrência em relação ao desconforto causado aos pedestres.

As vibrações induzidas por pessoas podem conduzir as estruturas ao estado limite de serviço, pois, o corpo humano é muito sensível às vibrações, segundo Wright e Green (1959) e Smith (1988). É muito improvável que essas vibrações causem dano à estrutura, fato confirmado por vários casos relatados na literatura a respeito das vibrações em passarelas, nas quais, apesar das vibrações excessivas, não ocorreram danos na estrutura. Entretanto, deve ser reforçado que outras ações decorrentes de distúrbios ou atos de vandalismo podem causar riscos para a integridade das estruturas.
As vibrações em passarelas têm sido tratadas com atenção em vários códigos normativos de países desenvolvidos. Porém, existem muitas divergências na adoção dos limites para as vibrações excessivas, o que caracteriza uma indicação da incerteza de continuidade nos procedimentos de resolução dos problemas de vibrações induzidos por pedestres. Alguns códigos, como BS 5400 (BRITISH STANDARDS, 1978), SIA 160 (SWISS NATIONAL STANDARDS, 1989), OHBDC (ONTARIO HIGHWAY BRIDGE DESIGN CODE, 1991), CEB (COMITÉ EURI-INTERNATIONAL DU BÉTON, 1993), Eurocode 5 (EUROPEAN COMMITTEE FOR STANDARDIZATION, 1995), AASHTO (AMERICAN ASSOCIATION OF STATES HIGHTWAY AND TRANSPORTATION OFFICIALS, 1997), Eurocode 5 (EUROPEAN COMMITTEE FOR STANDARDIZATION, 2004), Bro (2004) e ISO 10137 (INTERNATIONAL ORGANIZATION FOR STANDARDIZATION, 2005), apresentam diretrizes detalhadas que incluem valores limites para frequências e picos de acelerações. Tais códigos possuem procedimentos de cálculos que são baseados na definição dos carregamentos de pedestres, todavia há discrepâncias nas acelerações calculadas por meio deles e as medidas experimentais realizadas em passarelas, como foram relatadas por Eyre e Cullington (1985).

Os presentes avanços nos instrumentos de medidas têm facilitado as investigações do comportamento dinâmico das estruturas, permitindo a obtenção das frequências naturais, modos de vibrar e taxa de amortecimento das estruturas. As técnicas de análise modal são baseadas em teorias já estabelecidas e assumem um comportamento linear das estruturas. Geralmente, os problemas investigados relativos a passarelas consideram que as mesmas possuem vibrações de pequena amplitude são caracterizados por baixa frequência. No entanto, ainda há poucas experiências na aplicação dessas técnicas em ensaios de passarelas.

O desenvolvimento de novas ferramentas experimentais para avaliar, de maneira precisa, as características estáticas e dinâmicas dos novos projetos permitiu a evolução do cálculo estrutural e, consequentemente, a construção de estruturas mais complexas e ambiciosas. Essas ferramentas proveram dados confiáveis para calibrar, atualizar e validar os modelos numéricos da análise estrutural usada no estágio de projeto. O contínuo envelhecimento e a subsequente deterioração estrutural de muitas estruturas existentes encorajaram o desenvolvimento de eficientes técnicas de detecção, com o objetivo de monitorar as estruturas ao longo do tempo. 
A literatura técnica internacional revela que este assunto é bastante atual e encontra-se em fase de desenvolvimento. A utilização de uma passarela pode deixar de ser normal devido às vibrações excessivas, caso ocorram deformações excessivas imediatas e ao longo do tempo que possam causar danos na estrutura. As vibrações, quando são excessivas, também provocam consequências sérias, tais como: interrupção do uso, reformas com custo elevado, desgaste da imagem dos profissionais envolvidos e perda de valor econômico do patrimônio.

O objetivo deste trabalho é apresentar uma revisão bibliográfica que possibilite um melhor entendimento dos tipos de carregamentos dinâmicos induzidos pelo movimento de pedestres em passarelas, explicando a ocorrência das vibrações nas direções transversal vertical e transversal horizontal.

\section{Carregamentos Induzidos por Pedestres}

Durante a caminhada em uma estrutura, os pedestres introduzem forças dinâmicas que variam no tempo. Essas forças têm componentes (nas direções transversal vertical, transversal horizontal e longitudinal) dependentes de parâmetros, tais como: frequência de passo, velocidade de caminhada e comprimento de passo. Ao longo dos anos, vários estudos foram realizados para quantificar as forças de caminhada. Inicialmente, os estudos deram mais atenção à componente na direção transversal vertical, sendo que quase todos os problemas documentados a respeito das vibrações induzidas por pedestres foram associados a essa componente.

Pesquisadores como Galbraith e Barton (1970) realizaram estudos relacionados aos modos de vibrar da força nas direções transversal vertical e transversal horizontal, alterando a velocidade dos passos. Concluíram que a componente da força na direção transversal vertical tem apenas um pico no caso de corrida e dois picos no caso de caminhada, Figura 1. A frequência dos passos e peso das pessoas foi identificada como fatores que ampliam os picos da componente nesta direção, enquanto o tipo de calçado e o tipo da superfície de caminhada não apresentaram influência. Durante a caminhada, há alguns curtos períodos em que ambos os pés estão no chão, ocorrendo uma sobreposição das forças de caminhada conforme Figura 1; por outro lado, na corrida há períodos em que ambos os pés estão fora do piso, conduzindo a zero a força registrada, Figura 1.

Uma grande pesquisa compreendida dentro das forças humanas relevantes para excitação das passarelas foi conduzida por Wheeler $(1980,1982)$, que sistematizou
Figura 1 - Padrão típico de forças para corrida e caminhada na direção transversal vertical.

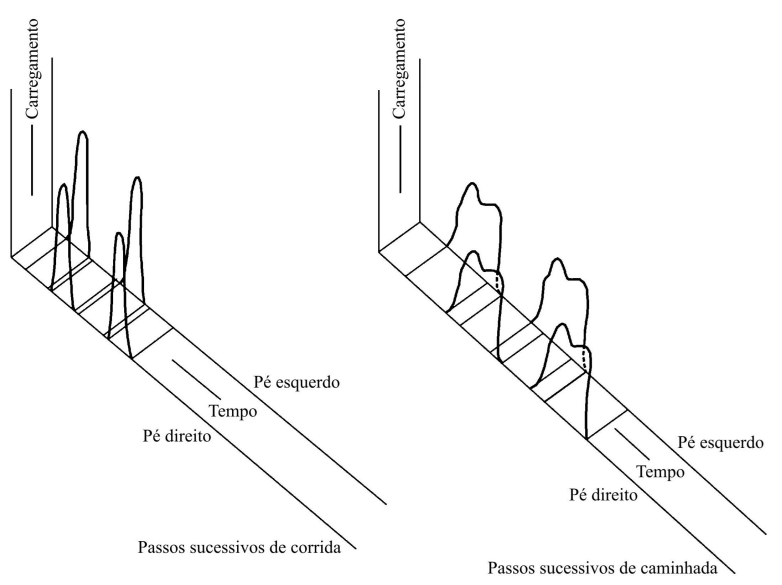

Fonte: Galbraith e Barton (1970).

o trabalho de outros pesquisadores relacionando os diferentes modos de movimento de caminhada humana, conforme Figura 2. O principal fator analisado nesse estudo foi verificar a influência da velocidade de passo na componente das várias formas da força de caminhada na direção transversal vertical.

Figura 2 - Forças na direção transversal vertical para diferentes forma de caminhada.
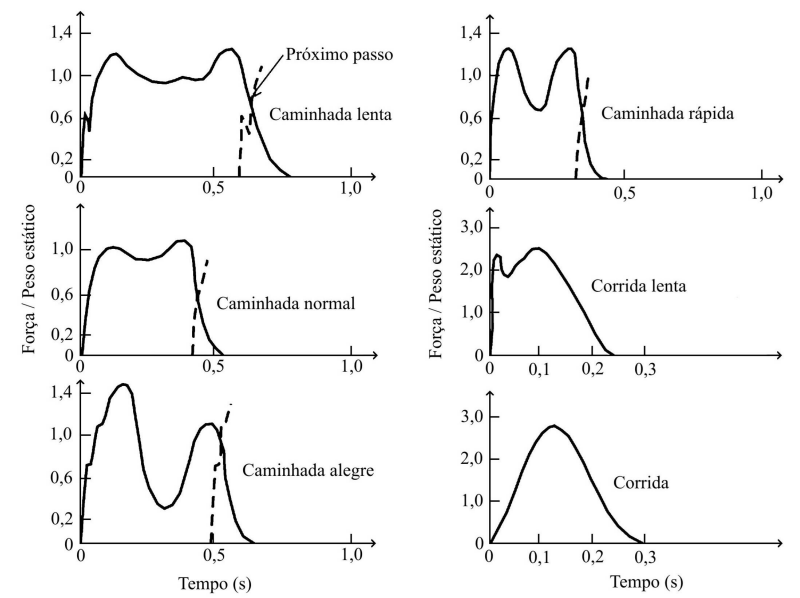

Fonte: Wheeler (1982).

Wheeler (1982) fez uma sistematização dos trabalhos mostrando que há uma dependência entre os muitos parâmetros de caminhada, como comprimento de passo, velocidade de movimento, picos de força e tempo de contato (tempo enquanto um dos pés está em contato com o piso), Figura 3. Notou-se que todos estes parâmetros são diferentes para diferentes pessoas, mas algumas conclusões gerais podem ser esboçadas. Por exemplo, com aumento da frequência de passo, o pico de amplitude, o comprimento de passo e a velocidade aumentam enquanto o tempo de contato diminui. Em certo momento, não mais ocorre o aumento do comprimento de passo e apenas a frequên- 
cia de passo e a velocidade aumentam de intensidade. $\mathrm{O}$ mesmo efeito ocorre quando a frequência aumenta e o tempo de contato permanece inalterado. Na Figura 3 são mostrados os gráficos obtidos por meio de correlações desses parâmetros, que foram sistematizados por Wheeler (1982).

Figura 3 - Dependência do comprimento do passo $\left(l_{p}\right)$, velocidade de passo $\left(v_{p}\right)$, pico de força versus peso estático $(c i)$ e tempo de contato $(t c)$ em diferentes frequências de passo $\left(f_{p}\right)$.
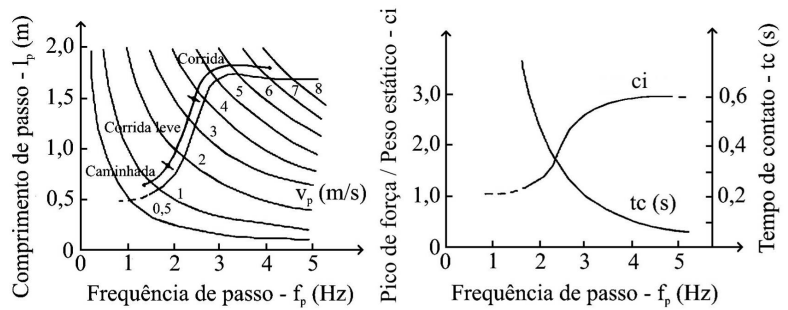

Fonte: Wheeler (1982)

As medidas da força de passo de indivíduos foram feitas pelos mais avançados e informativos sistemas de medidas contínuas no tempo, compreendendo vários tipos de caminhada. Para este propósito, Blanchard, Davies e Smith (1977) usaram uma máquina de andar descrita por Skorecki (1966); Rainer, Pernica e Allen (1988) usaram uma faixa de piso, enquanto Ebrahimpour et al. (1994, 1996) usaram uma plataforma instrumentada com várias placas de força. As medidas no tempo foram obviamente próximas, com o período (médio) igual ao recíproco valor da frequência (média) de passo. Infelizmente, em todos estes trabalhos foi dada maior atenção para obter somente as forças na direção transversal vertical. Embora baseado nas medidas de Andriacchi, Ogle e Galante (1977) e levando em consideração que a frequência fundamental da força na direção transversal horizontal induzida pela caminhada é duas vezes menor do que a frequência fundamental da força nas direções transversal vertical e longitudinal, geralmente podem ser construídos os modos contínuos para força em todas as três direções, assumindo uma perfeita periodicidade, segundo Bachmann e Ammann (1987), conforme Figura 4.

Uma confiável descrição estatística da frequência de caminhada normal foi dada por Matsumoto et al. (1972, 1978), que investigaram um exemplo de 505 pessoas e concluíram que as frequências seguem uma distribuição normal com uma média de passo igual a 2,0 Hz e desvio padrão igual a 0,173 Hz, conforme Figura 5. Kerr e Bishop (2001) obtiveram uma frequência média de 1,9 Hz, mas de uma investigação de somente 40 sujeitos. Compreensão estatística similar baseada em investigações, como a
Figura 4 - Registro periódico das forças nas direções transversal vertical, transversal horizontal e longitudinal de pessoas caminhando ao longo do tempo.
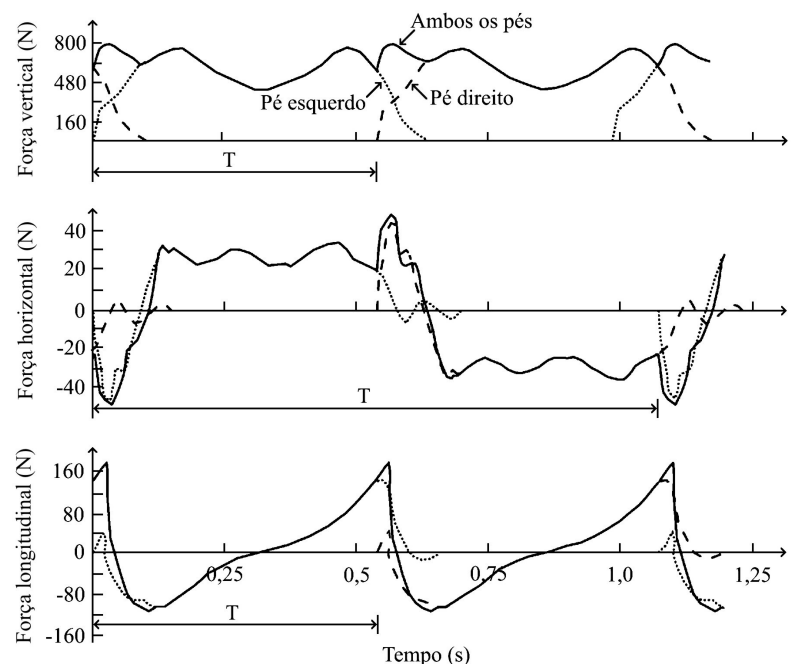

Fonte: Bachmann e Ammann (1987).

dada por Matsumoto et al. (1978) para caminhada, não existe para outro tipo de força induzida por pedestres. Porém existem algumas propostas sobre as faixas típicas de frequência para diferentes atividades humanas (correr, pular, saltar etc.). Por exemplo, Bachmann, Pretlove e Rainer (1995) definiram tipicamente a variação de frequências na direção transversal vertical de 1,6 a $2,4 \mathrm{~Hz}$ para caminhada; 2,0 a 3,5 Hz para corrida; 1,8 a 3,4 Hz para pular; 1,5 a 3,0 Hz para saltar e 0,4 a $0,7 \mathrm{~Hz}$ para o balanço na direção transversal horizontal do corpo enquanto estacionário.

Figura 5 - Distribuição normal da frequência de caminhada na direção transversal vertical.

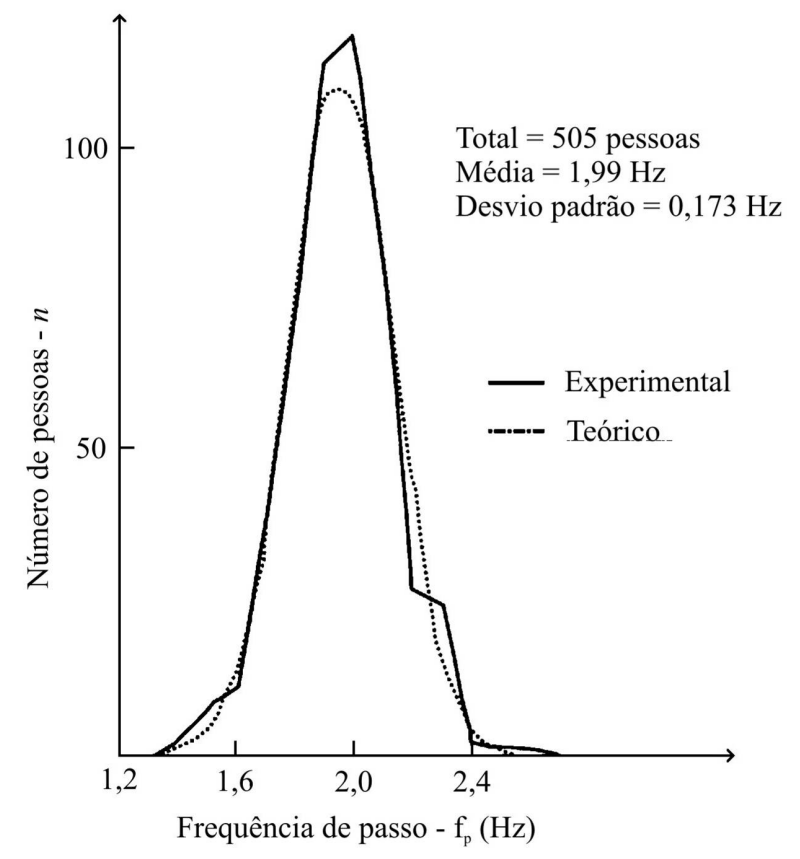

Fonte: Matsumoto et al. (1972). 
Na Figura 6 têm-se as frequências típicas da função de força na direção transversal vertical, segundo Wheeler (1982), de caminhada lenta a rápida. As funções de força mostradas na Figura 6 são dependentes da frequência de passo e as atividades de caminhada classificadas em quatro tipos de frequência de passo que corresponde a um tipo de atividade. Neste caso, estabeleceram-se as faixas de frequências para uma função similar de força na direção que identifica caminhada lenta (menor do que $1,8 \mathrm{~Hz}$ ), caminhada normal (1,8 a 2,2 Hz), caminhada alegre (2,2 a $2,7 \mathrm{~Hz}$ ) e caminhada rápida (maior do que 2,7 Hz).

Figura 6 - Padrão típico de forças de caminhada na direção transversal vertical.

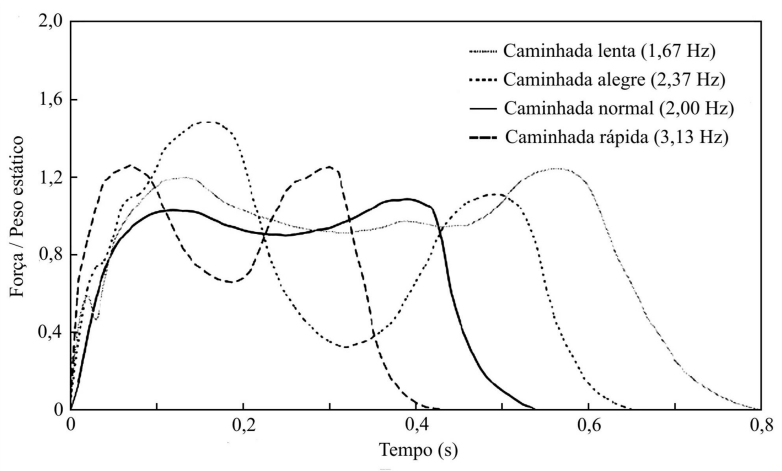

Fonte: Wheeler (1982).

Como foi visto no início deste item, o tráfego de pedestres produz forças dinâmicas variantes no tempo, com componentes nas direções transversal vertical, transversal horizontal e longitudinal. Isso decorre do simples caminhar de pedestres que produz forças devido à aceleração e à desaceleração da massa do corpo. A frequência típica de passo para caminhada, em torno de 2 passos por segundo, origina uma força na direção transversal vertical com 2,0 $\mathrm{Hz}$ de frequência. A caminhada lenta está na faixa de 1,4 a $1,7 \mathrm{~Hz}$ e a caminhada rápida na faixa de 2,2 a 2,4 Hz. Isto significa que a faixa total da frequência de força na direção transversal vertical é 1,4 a 2,4 Hz, com uma média de 2,0 Hz. Como a componente na direção transversal horizontal da força é aplicada em metade da frequência de passo, a frequência da força nesta direção está na faixa de 0,7 a 1,2 Hz (Figura 7), segundo Bachmann (2002). Muitas passarelas têm frequência nas direções transversal vertical e transversal horizontal dentro dos limites mencionados acima (frequências nas direções transversal vertical de 1,4 a 2,4 Hz e transversal horizontal de 0,7 a 1,2 Hz). Portanto, estas têm um grande potencial de vir a sofrerem vibrações excessivas devido à ação de pedestres.
Figura 7 - Faixas de frequências nas direções transversal vertical e transversal horizontal em passarelas.

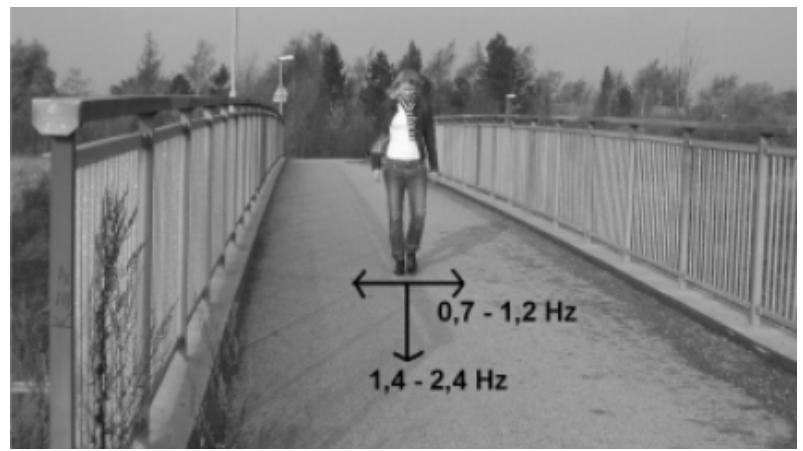

Fonte: Nakamura (2002).

\section{Mecanismo de vibração em passarelas, segundo Fujino et al. (1993)}

O carregamento dinâmico do movimento de caminhada humana é, em sua maior parte, na direção transversal vertical. A frequência predominante para essa componente é, aproximadamente, $2,0 \mathrm{~Hz}$, embora varie ligeiramente dependendo do indivíduo e do tipo de caminhada, segundo Matsumoto et al. (1978), Bachmann e Ammann (1987). Consequentemente, se uma passarela qualquer tem uma frequência natural próxima de $2,0 \mathrm{~Hz}$, para um modo de vibrar na direção transversal vertical, essa pode potencialmente ser induzida pelos pedestres, dando origem ao fenômeno da ressonância. Esse tipo de fenômeno, que pode causar notáveis amplitudes, ocorreu em muitas passarelas. Para evitar tais vibrações na direção transversal vertical, as especificações de projeto para passarelas no Japão requerem que as frequências naturais do modo de vibrar desta direção devem estar fora da faixa de 1,5 a 2,3 $\mathrm{Hz}$.

Os movimentos humanos não estão diretamente na direção transversal vertical de caminhada, pois o ser humano caminha trocando seu peso periodicamente de uma perna para outra. Na verdade, sempre caminha seguindo um movimento na direção transversal horizontal de ziguezague do corpo, cuja frequência dominante é igual a 1 $\mathrm{Hz}$.

A pesquisa realizada por Fujino et al. (1993) descreveu as vibrações nas direções transversal vertical e transversal horizontal induzidas por pedestres em uma passarela sob um extremo congestionamento. As observações mostraram evidências de sincronismo da ação de caminhada de muitos pedestres durante a vibração na direção transversal horizontal da passarela.

A passarela estudada nessa pesquisa por Fujino et al. (1993) foi construída em sistema estaiado com dois vãos contínuos em viga caixão de aço, conforme Figura 8, e 
com dois planos de estais. Tem um vão total de $179 \mathrm{~m} \mathrm{e}$ largura da via de pedestres igual a 5,25 m.

Figura 8 - Vista na direção longitudinal (a) e seção transversal da viga caixão de aço (b) da passarela em sistema estaiado.

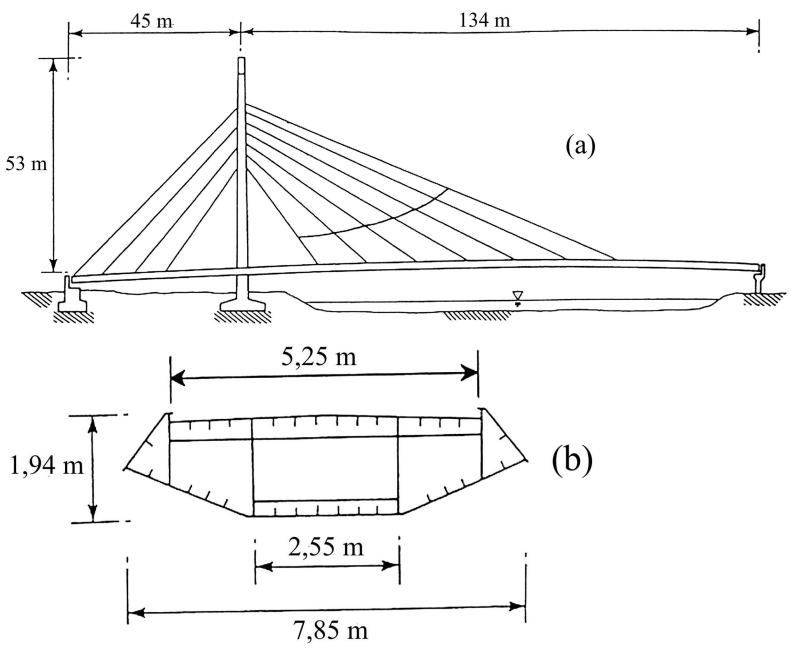

Fonte: Fujino et al. (1993).

A passarela é adjacente a um estádio de competição de barcos e conecta o estádio a um terminal de ônibus. Depois de uma grande competição de barcos, mais de 20.000 pessoas passaram algumas vezes sobre a passarela, dentro de um intervalo de tempo de $20 \mathrm{~min}$, ocorrendo congestionamento com mais ou menos 2.000 pedestres caminhando simultaneamente na passarela. Sob esta situação, além da vibração na direção transversal vertical, também foi observada notável vibração na direção transversal horizontal da passarela. A primeira, segunda e terceira frequência natural do modo na direção transversal vertical da passarela são $0,7,1,4$ e 2,0 Hz, respectivamente, enquanto a primeira frequência natural na direção transversal horizontal da viga é $0,9 \mathrm{~Hz}$, todas confirmadas por medidas experimentais. Alguns cabos também tinham sua primeira frequência de vibração natural na faixa de 0,9 a $1 \mathrm{~Hz}$ e vibraram com grandes amplitudes.

No estudo das vibrações ocorridas nessa passarela, dois tipos de mecanismo de vibração foram pensados por Fujino et al. (1993). Um correspondente à excitação na direção transversal vertical da viga de frequência natural 2,0 $\mathrm{Hz}$, que é ressonantemente excitada devido à caminhada humana (frequência de $2 \mathrm{~Hz}$ ); esse movimento na direção transversal vertical também provocou o movimento na direção transversal horizontal de alguns cabos, na frequência $1 \mathrm{~Hz}$, conforme Figura 9.

O outro mecanismo é a ressonância tipo linear direta, pela qual a força na direção transversal horizontal, com frequência de 1,0 Hz devido à caminhada humana, fornece
Figura 9 - Mecanismo de vibração na direção transversal vertical.
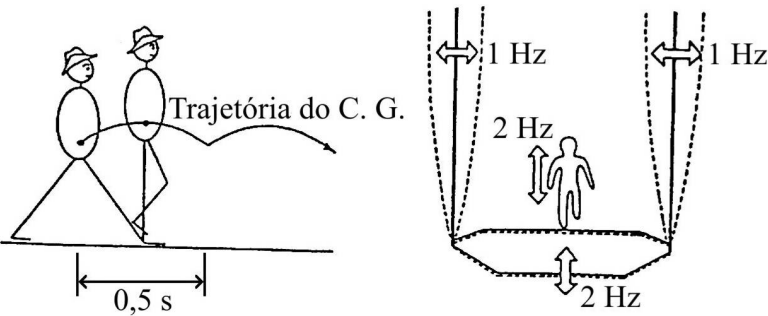

Fonte: Fujino et al. (1993).

uma força diretamente para o modo de vibrar na direção transversal horizontal da viga de frequência natural igual a $0,9 \mathrm{~Hz}$ e para alguns cabos cujas frequências estão próximas de 0,9 Hz. Segundo Fujino et al. (1993), as forças horizontais produzidas devido ao caminhar das pessoas são decorrentes das oscilações horizontais do movimento do centro de gravidade do corpo, conforme Figura 10, e são transferidas para as estruturas por meio do contato da superfície. Essas oscilações horizontais do corpo humano são da ordem de 1 a $2 \mathrm{~cm}$ com uma frequência de aproximadamente $1 \mathrm{~Hz}$ na direção transversal horizontal, conforme Figura 10. No entanto, as verificações relacionadas aos parâmetros horizontais não estão bem definidas, pois a maioria dos trabalhos tem sido feitos superficialmente.

Figura 10 - Mecanismo de vibração na direção transversal horizontal.
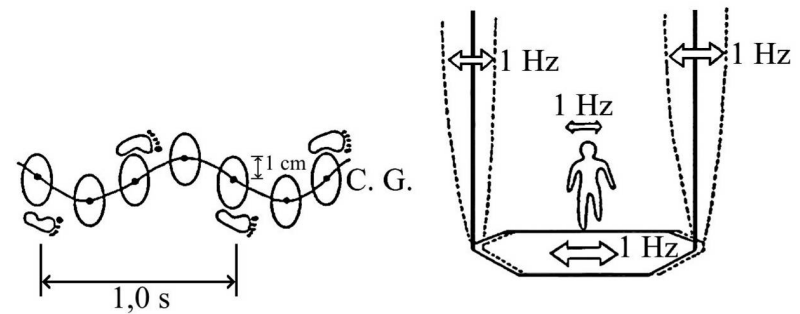

Fonte: Fujino et al. (1993).

\section{Modelagem da Força}

Na literatura são encontrados modelos de carregamentos dinâmicos aplicados no domínio do tempo e no domínio da frequência. Este trabalho estudará apenas a respeito de modelos no domínio do tempo. Geralmente, dois tipos de modelos no domínio do tempo têm sido encontrados na literatura: determinístico e probabilístico. O primeiro estabelece um modelo geral de força para cada tipo de atividade humana; o segundo leva em conta alguns parâmetros que influenciam na força humana, como a frequência da atividade e peso das pessoas. Este trabalho apresentará 
apenas o modelo determinístico de força que é relevante para o entendimento desse assunto.

O modelo determinístico no domínio do tempo para o movimento de caminhar e correr considera como se ambos os pés produzissem exatamente a mesma força periódica para esses casos. A suposição de repetições perfeitas é usada na geração da modelagem dos carregamentos destrutivos por uma simples pessoa e grupos pequenos de pessoas.

No sentido de estabelecer uma modelagem das forças dinâmicas de carregamentos, os pesquisadores Blanchard, Davies e Smith (1977) propuseram um modelo simples de força de caminhada que toma como base o fenômeno da ressonância devido somente ao primeiro harmônico, que possui fator dinâmico de carregamento igual a 0,257 para um peso de pedestre igual a $700 \mathrm{~N}$. Este foi indicado para passarelas com frequência fundamental na direção transversal vertical de até $4 \mathrm{~Hz}$; nas frequências naturais entre 4 e $5 \mathrm{~Hz}$, alguns fatores de redução foram aplicados, considerando as baixas amplitudes do segundo harmônico, pois esta faixa de frequência pode não ser excitada pelo primeiro harmônico de caminhada. Os resultados e conclusões referentes ao seguinte estudo foram introduzidos no código britânico BS 5400 (BRITISH STANDARDS, 1978) para serem utilizados no dimensionamento de passarelas e, dessa forma, dar segurança aos usuários.

Os pesquisadores Bachmann e Ammann (1987) foram pioneiros em informar os primeiros cinco harmônicos para força de caminhada nas direções transversal vertical, transversal horizontal e longitudinal dos modelos de carregamento determinístico no domínio do tempo. Mostraram que o primeiro e o terceiro harmônico da força na direção transversal horizontal e o primeiro e segundo harmônico da força na direção longitudinal são dominantes, Figura 11. É interessante que foram identificados

Figura 11 - Componentes harmônicos da força de caminhada nas direções (a) transversal vertical, (b) transversal horizontal e (c) longitudinal.

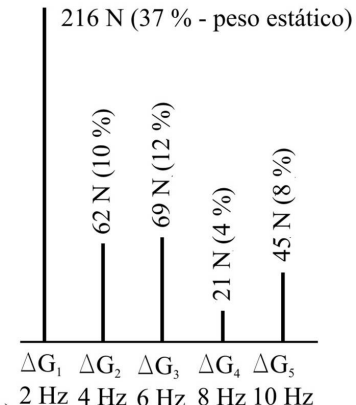$$
\text { Z Z }
$$

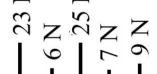

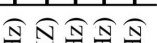$$
\text { (a) }
$$$$
2 \mathrm{~Hz} 4 \mathrm{~Hz} 6 \mathrm{~Hz} 8 \mathrm{~Hz} 10 \mathrm{~Hz}
$$

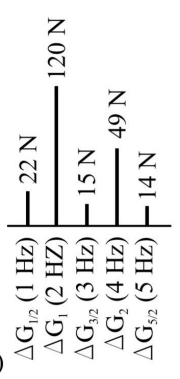

alguns sub-harmônicos nas direções transversal horizontal e longitudinal, conforme mostrado na Figura 11, que ocorreram como uma consequência do maior pronunciamento da batida dos pés na direção transversal horizontal.

No final dos anos 1980 um aumento significante neste campo foi provido por um excelente trabalho de Rainer et al. (1988). Estes mediram contínuas forças de uma simples pessoa não somente caminhando, mas também correndo e pulando, e confirmaram que os fatores de carregamento dinâmico $\left(\alpha_{i}\right)$ dependem fortemente da frequência das atividades. Na Figura 12 estão representadas as curvas no domínio da frequência para os quatro primeiros fatores dinâmicos de carregamento $\left(\alpha_{i}\right)$ na direção transversal vertical para as forças de caminhada, corrida e pulo. Atualmente muitos pesquisadores, tomando como base a decomposição de Fourier, quantificaram os fatores dinâmicos de carregamento $\left(\alpha_{i}\right)$ que são as bases para estes modelos de periodicidade perfeita de forças induzidas por humanos. Na Tabela 1, têm-se os fatores dinâmicos de carregamento estimados por diferentes autores para o modelo de força de um indivíduo executando diferentes atividades nas direções transversal vertical, transversal horizontal e longitudinal.

Figura 12 - Fatores de carregamento dinâmicos $\left(\alpha_{i}\right)$ na direção transversal vertical para os quatro primeiros harmônicos da força de caminhada (a), corrida (b) e pulo (c).

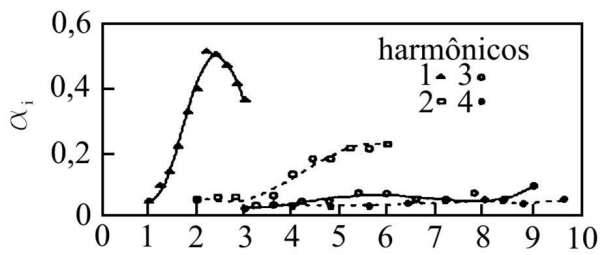

(a) Frequência de passo $-\mathrm{f}_{\mathrm{p}}(\mathrm{Hz})$

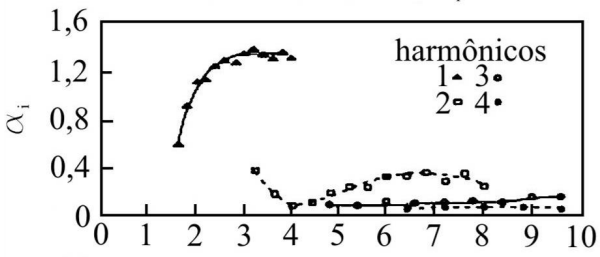

(b) Frequência de passo $-\mathrm{f}_{\mathrm{p}}(\mathrm{Hz})$

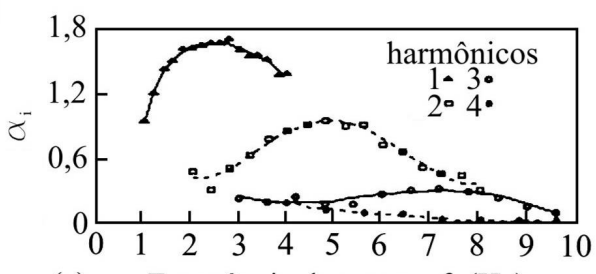

(c) Frequência de passo $-\mathrm{f}_{\mathrm{p}}(\mathrm{Hz})$

Fonte: Rainer, Pernica e Allen (1988).

Fonte: Bachmann e Ammann (1987). 
Segundinho, P. G. A. ${ }^{1}$; Dias, A. A. ${ }^{2}$; Carreira, M. R. ${ }^{3}$

Tabela 1 - Fatores dinâmicos de carregamento estimado por diferentes autores

\begin{tabular}{|c|c|c|c|}
\hline Autores & $\begin{array}{l}\text { Fatores dinâmicos } \\
\text { de carregamento } \alpha_{i}\end{array}$ & Comentário & Atividade-direção \\
\hline Blanchard (1977) & $\alpha_{1}=0,257$ & $\begin{array}{c}\text { Fator dinâmico de } \\
\text { carregamento é redutor } \\
\text { para frequências de } 4 \text { a } 5 \mathrm{~Hz}\end{array}$ & $\begin{array}{l}\text { Caminhada- } \\
\text { transversal vertical }\end{array}$ \\
\hline $\begin{array}{c}\text { Bachmann e } \\
\text { Ammann (1987) }\end{array}$ & $\begin{array}{l}\alpha_{1}=0,4-0,5 \\
\alpha_{2}=\alpha_{3}=0,1\end{array}$ & $\begin{array}{c}\text { Entre } 2 \text { e } 2,4 \mathrm{~Hz}, \\
\text { aproximadamente } 2 \mathrm{~Hz}\end{array}$ & $\begin{array}{l}\text { Caminhada- } \\
\text { transversal vertical }\end{array}$ \\
\hline $\begin{array}{l}\text { Schulze (depois } \\
\text { de Bachmann e } \\
\text { Ammann (1987)) }\end{array}$ & $\begin{array}{c}\alpha_{1}=0,37 ; \alpha_{2}=0,10 \\
\alpha_{3}=0,12 ; \alpha_{4}=0,04 \\
\alpha_{5}=0,08\end{array}$ & $\mathrm{Em} 2 \mathrm{~Hz}$ & $\begin{array}{l}\text { Caminhada- } \\
\text { transversal vertical }\end{array}$ \\
\hline $\begin{array}{l}\text { Schulze(depois } \\
\text { de Bachmann e } \\
\text { Ammann (1987)) }\end{array}$ & $\begin{array}{c}\alpha_{1}=0,039 ; \alpha_{2}=0,010 \\
\alpha_{3}=0,043 ; \alpha_{4}=0,012 \\
\alpha_{5}=0,015\end{array}$ & $\mathrm{Em} 2 \mathrm{~Hz}$ & $\begin{array}{l}\text { Caminhada- } \\
\text { transversal horizontal }\end{array}$ \\
\hline $\begin{array}{l}\text { Schulze (depois } \\
\text { de Bachmann e } \\
\text { Ammann (1987)) }\end{array}$ & $\begin{array}{c}\alpha_{1 / 2}=0,037 ; \alpha_{1}=0,204 \\
\alpha_{3 / 2}=0,026 ; \alpha_{2}=0,083 \\
\alpha_{5 / 2}=0,024\end{array}$ & $\mathrm{Em} 2 \mathrm{~Hz}$ & $\begin{array}{l}\text { Caminhada- } \\
\text { longitudinal }\end{array}$ \\
\hline $\begin{array}{l}\text { Rainer et } \\
\text { al. (1988) }\end{array}$ & $\alpha_{1}, \alpha_{2}, \alpha_{3}$ e $\alpha_{4}$ & $\begin{array}{c}\text { Fatores dinâmicos de } \\
\text { carregamento dependentes } \\
\text { das frequências, Fig. } 4.12\end{array}$ & $\begin{array}{c}\text { Caminhada, corrida, } \\
\text { pulo-transversal } \\
\text { vertical }\end{array}$ \\
\hline $\begin{array}{l}\text { Bachmann et } \\
\text { al. (1995) }\end{array}$ & $\begin{array}{c}\alpha_{1}=0,4 / 0,5 \\
\alpha_{2}=, \alpha_{3}=0,1 /-\end{array}$ & $\operatorname{Em} 2,0 / 2,4 \mathrm{~Hz}$ & $\begin{array}{l}\text { Caminhada- } \\
\text { transversal vertical }\end{array}$ \\
\hline $\begin{array}{l}\text { Bachmann et } \\
\text { al. (1995) }\end{array}$ & $\alpha_{1}=\alpha_{3}=0,1$ & $\mathrm{Em} 2,0 \mathrm{~Hz}$ & $\begin{array}{l}\text { Caminhada- } \\
\text { transversal horizontal }\end{array}$ \\
\hline $\begin{array}{l}\text { Bachmann et } \\
\text { al. (1995) }\end{array}$ & $\begin{array}{c}\alpha_{1 / 2}=0,1 ; \alpha_{1}=0,2 \\
\alpha_{2}=0,1\end{array}$ & $\mathrm{Em} 2,0 \mathrm{~Hz}$ & $\begin{array}{l}\text { Caminhada- } \\
\text { longitudinal }\end{array}$ \\
\hline $\begin{array}{l}\text { Bachmann et } \\
\text { al. (1995) }\end{array}$ & $\begin{array}{c}\alpha_{1}=1,6 ; \alpha_{2}=0,7 \\
\alpha_{3}=0,2\end{array}$ & Em $2,0-3,0 \mathrm{~Hz}$ & $\begin{array}{l}\text { Corrida- } \\
\text { transversal vertical }\end{array}$ \\
\hline Young (2001) & $\begin{array}{c}\alpha_{1}=0,37(f-0,95) \leq 0,5 \\
\alpha_{2}=0,054+0,0044 f \\
\alpha_{3}=0,026+0,0050 f \\
\alpha_{4}=0,010+0,0051 f\end{array}$ & $\begin{array}{l}\text { Estes são valores médios } \\
\text { para fatores dinâmicos } \\
\text { de carregamento }\end{array}$ & $\begin{array}{c}\text { Caminhada- } \\
\text { transversal vertical }\end{array}$ \\
\hline $\begin{array}{l}\text { Bachmann et } \\
\text { al. (1995) }\end{array}$ & $\begin{array}{c}\alpha_{1}=1,8 / 1,7 ; \alpha_{2}=1,3 / 1,1 \\
\alpha_{3}=0,7 / 0,5\end{array}$ & $\begin{array}{c}\text { Pulo normal } \\
\text { em } 2,0 / 3,0 \mathrm{~Hz}\end{array}$ & $\begin{array}{l}\text { Pulo-transversal } \\
\text { vertical }\end{array}$ \\
\hline $\begin{array}{l}\text { Bachmann et } \\
\text { al. (1995) }\end{array}$ & $\begin{array}{c}\alpha_{1}=1,9 / 1,8 ; \alpha_{2}=1,6 / 1,3 \\
\alpha_{3}=1,1 / 0,8\end{array}$ & $\begin{array}{c}\text { Pulo alto } \\
\text { em } 2,0 / 3,0 \mathrm{~Hz}\end{array}$ & $\begin{array}{l}\text { Pulo-transversal } \\
\text { vertical }\end{array}$ \\
\hline $\begin{array}{l}\text { Bachmann et } \\
\text { al. (1995) }\end{array}$ & $\begin{array}{c}\alpha_{1}=0,17 / 0,38 ; \alpha_{2}=0,10 / 0,12 \\
\alpha_{3}=0,04 / 0,02\end{array}$ & $\begin{array}{c}\text { Em 1,6/2,4 } \\
\mathrm{Hz}\end{array}$ & $\begin{array}{c}\text { Salto-transversal } \\
\text { vertical }\end{array}$ \\
\hline $\begin{array}{l}\text { Bachmann et } \\
\text { al. (1995) }\end{array}$ & $\alpha_{1}=0,5$ & Em $0,6 \mathrm{~Hz}$ & $\begin{array}{l}\text { Corpo balançando } \\
\text { enquanto parado- } \\
\text { transversal horizontal }\end{array}$ \\
\hline $\begin{array}{l}\text { Yao et al. } \\
\text { (2002) }\end{array}$ & $\alpha_{1}=0,7 ; \alpha_{2}=0,25$ & $\begin{array}{c}\text { Salto livre em uma } \\
\text { plataforma flexível com } \\
\text { frequência natural de } 2,0 \mathrm{~Hz}\end{array}$ & $\begin{array}{l}\text { Salto-transversal } \\
\text { vertical }\end{array}$ \\
\hline
\end{tabular}

Fonte: Autores. 


\section{Modelos de carregamentos}

As análises de cada ação dinâmica produzida pelos pedestres indicam que as forças dinâmicas de caminhada, corrida e pulo $F(t)$ podem ser representadas em um período T. Isto é, as componentes dinâmicas da força de atividade podem ser representadas por um termo e pela somatória de termos, que é uma série de Fourier com fatores dinâmicos de carregamento $\left(\alpha_{i}\right)$ nas frequências discretas $(i \cdot f)$.

Inicialmente, foi sugerido por Bachmann e Ammann (1987) um modelo de representação dinâmica dos carregamentos composto pela parcela estática correspondente ao peso do pedestre e uma combinação de harmônicos associados à excitação dinâmica. A força que representa o movimento de caminhar humano pode ser obtida por meio da Equação (1) e as componentes dinâmicas da força de caminhada são representadas por uma série de Fourier. O seguinte modelo de carregamento dinâmico possibilita que a posição do carregamento dinâmico seja alterada de acordo com a posição do pedestre na estrutura, isto é, a função gerada tem uma descrição temporal e espacial.

$$
F(t)=P\left(1+\sum_{i=1}^{N} \alpha_{i} \cdot \sin \left(i \cdot 2 \pi \cdot f_{p} \cdot t+\varphi_{i}\right)\right)
$$

em que:

$P$ : peso do pedestre tomado igual a $700 \mathrm{~N}$;

$\alpha_{i}$ : coeficiente dinâmico para o ith harmônico, Tabela 1; $f_{p}$ : frequência de passo $(\mathrm{Hz})$;

$t$ : tempo de percurso do carregamento $(s)$;

$\varphi_{i}$ : ângulo de fase do ith harmônico da força, conforme mostrado na Tabela 2;

$i$ : número de ordem do harmônico;

$N$ : número total de harmônicos contribuintes.

Todavia, para um melhor entendimento desse modelo faz-se necessário entender outros parâmetros desta modelagem que estão associados à frequência de passo, como a distância e a velocidade de passo. No estudo realizado por Bachmann e Ammann (1987), a excitação dinâmica associada ao caminhar do ser humano sobre a passarela foi obtida mediante o emprego da Equação (1), por meio do uso de quatro harmônicos para gerar a função de carregamento. É bom lembrar que uma série de Fourier necessita dos fatores dinâmicos de carregamento $\left(\alpha_{i}\right)$, uma vez que representa a amplitude central dos harmônicos da força registrada e são normalizados pelo próprio peso do pedestre $(P)$. Apesar de o modelo ser eficiente, os trabalhos atuais de Figueiredo et al. (2008) utilizaram, com grande sucesso, o modelo sugerido por Murray et al. (1997).

Os modelos de forças considerados por Murray, Allen e Ungar (1997), em suas investigações, também indicam que as forças $F(t)$ de caminhada, corrida e pulo podem ser representadas para um período $\mathrm{T}$, por apenas um harmônico ressonante de carga, Equação (2), ou por meio de uma série de Fourier, Equação (3), sendo ambos aplicados no ponto de maior amplitude modal da passarela. Na Equação (2) não há uma variação espacial da carga no decorrer do tempo, pois a mesma é aplicada sempre no meio do vão, o que de fato não acontece durante o movimento de pedestre sobre a passarela. Porém, o modelo de carregamento dinâmico representado pela Equação (3) pode ser adotado tanto para uma variação espacial e temporal sobre a passarela, quanto para uma variação temporal aplicada no meio do vão. Este também é composto pela parcela estática correspondente ao peso do pedestre e uma combinação de harmônicos associados à excitação dinâmica por meio dos fatores dinâmicos de carregamento $\left(\alpha_{i}\right)$, conforme mostrado na Tabela 2.

$$
F(t)=P \cdot \alpha_{i} \cdot \cos \left(i \cdot 2 \pi \cdot f_{p} \cdot t\right)
$$

$$
F(t)=P\left(1+\sum_{i=1}^{N} \alpha_{i} \cdot \cos \left(i \cdot 2 \pi \cdot f_{p} \cdot t+\varphi_{i}\right)\right)
$$

em que:

$P$ : peso do pedestre tomado igual a $700 \mathrm{~N}$;

$\alpha_{i}$ : coeficiente dinâmico para o ith harmônico, Tabela 2 ;

$f_{p}$ : frequência de passo $(\mathrm{Hz})$;

$t$ : tempo de percurso do carregamento $(s)$;

$\varphi_{i}$ : ângulo de fase do ith harmônico da força, conforme mostrado na Tabela 2;

$i$ : número de ordem do harmônico;

$N$ : número total de harmônicos contribuintes.

O modelo de carregamento mostrado a seguir foi proposto por Varela (2004), seguindo a metodologia dos modelos de carregamentos propostos por Bachmann e Ammann (1987) e Murray, Allen e Ungar (1997). No entanto, neste modelo é introduzido o efeito proveniente do impacto transiente do calcanhar, conforme mostrado na Equação (4). 
Tabela 2 - Fatores dinâmicos de carregamento.

\begin{tabular}{c|c|c|c|c|c|c|c|c|c}
\hline & \multicolumn{3}{|c|}{ Pessoa caminhando } & \multicolumn{3}{c|}{ Classes de aeróbica } & \multicolumn{3}{c}{ Grupos de dança } \\
\hline $\mathrm{i}$ & $\begin{array}{c}f_{p} \\
(\mathrm{~Hz})\end{array}$ & $\alpha_{i}$ & $\varphi_{i}$ & $f_{p}(\mathrm{~Hz})$ & $\alpha_{i}$ & $\varphi_{i}$ & $f_{p}(\mathrm{~Hz})$ & $\alpha_{i}$ & $\varphi_{i}$ \\
\hline 1 & $1,6-2,2$ & 0,5 & 0 & $2,0-2,75$ & 1,5 & - & $1,5-3,0$ & 0,5 & - \\
\hline 2 & $3,2-4,4$ & 0,2 & $\pi / 2$ & $4,0-5,5$ & 0,6 & - & - & - & - \\
\hline 3 & $4,8-6,6$ & 0,1 & $\pi / 2$ & $6,0-8,25$ & 0,1 & - & - & - & - \\
\hline 4 & $6,4-8,8$ & 0,05 & $\pi / 2$ & - & - & - & - & - & - \\
\hline
\end{tabular}

Fonte: Murray et al. (1997).

$$
\begin{aligned}
F(t)= & \left(\frac{f_{m i} F_{m}-P}{0,04 T_{P}}\right) t+P ; \text { se } 0 \leq t \leq 0,04 T_{P} \\
F(t)= & f_{m i} F_{m}\left[\frac{C_{1}\left(t-0,04 T_{P}\right)}{0,02 T_{P}}+1\right] ; \\
& \quad \text { se } 0,04 T_{P} \leq t \leq 0,06 T_{P} \\
F(t)= & F_{m} ; \operatorname{se} 0,06 T_{P} \leq t \leq 0,15 T_{P} \\
F(t)= & P+\sum_{i=1}^{N} P \cdot \alpha_{i} \sin \left[i \cdot 2 \pi \cdot f_{P}\left(t+0,1 T_{P}\right)+\varphi_{i}\right] ; \\
& \operatorname{se} 0,15 T_{P} \leq t \leq 0,90 T_{P} \\
F(t)= & 10\left(P-C_{2}\right)\left(\frac{t}{T_{P}}-1\right)+P \\
& \text { se } 0,04 T_{P} \leq t \leq 0,06 T_{P}
\end{aligned}
$$

em que:

$F_{m}$ : valor máximo da série de Fourier dado pela Equação (5);

$f_{m i}$ : fator de majoração do impacto do calcanhar, ou seja, é a relação entre o valor do pico transiente do calcanhar e o valor máximo da série de Fourier;

$f_{p}$ : frequência de passo $(\mathrm{Hz})$;

$T_{P}:$ tempo de passo $(s)$;

$t:$ tempo de carregamento $(s)$;

$\varphi_{i}$ : ângulo de fase do ith harmônico da força;

$i$ : número de ordem do harmônico;

$N$ : número total de harmônicos contribuintes;

$C_{1}$ : coeficiente dado pela Equação (6);

$C_{2}$ : coeficiente dado pelas Equações (7) e (8).

$$
\begin{aligned}
& F_{m}=P\left(1+\sum_{i=1}^{N} \alpha_{i}\right) \\
& C_{1}=\left(\frac{1}{f_{m i}}-1\right) \\
& C_{2}=P\left(1-\alpha_{2}\right) \text { se } N=3 \\
& C_{2}=P\left(1-\alpha_{2}+\alpha_{4}\right) \text { se } N=4
\end{aligned}
$$

Segundo Varela (2004), o fator de majoração de impacto do calcanhar recomendado é igual a 1,12 e os ângulos de fase são iguais a $\varphi_{1}=0, \varphi_{2}=\frac{\pi}{2}, \varphi_{3}=\pi$ e $\varphi_{4}=\frac{3 \pi}{2}$, porque, conforme exposto, as equações propostas foram concebidas utilizando tais valores. A Equação (4) apresentará singularidades e ficará descaracterizada caso sejam utilizados valores diferentes dos apresentados. Varela (2004) também afirma que nem sempre os três primeiros coeficientes da série de Fourier são suficientes para representar adequadamente as atividades humanas e afirma que o valor da frequência de harmônicos superiores, o quarto harmônico, por exemplo, quando próximo o suficiente de uma das frequências fundamentais da estrutura, faz com que a resposta da mesma seja amplificada consideravelmente devido ao fenômeno da ressonância provocada pelo movimento dos pedestres.

Nos estudos de Figueiredo (2005), concluiu-se que os modelos de carregamento propostos por Murray Allen e Ungar (1997) e Varela (2004), os quais incorporam a variação espacial e temporal da carga dinâmica, bem como os quatro harmônicos componentes da excitação, quando aplicados sobre os modelos estruturais analisados, fornecem respostas dinâmicas de intensidade bem superiores às obtidas pelo emprego dos modelos de carregamento de Murray, Allesn e Ungar (1997) que levam em conta somente a variação temporal de um carregamento dinâmico localizado na região central da passarela. Nesses estudos também se destacou que os resultados obtidos com base no emprego do modelo de carregamento proposto por Murray Allesn e Ungar (1997) apresentam valores bastante próximos do modelo de carregamento proposto por Varela (2004) e observou-se que o efeito do impacto do calcanhar é maior para estruturas com taxa de amortecimento menores do que $1 \%$. Com certeza, deve-se aproveitar a enorme potencialidade com uma variação maior dos seus coeficientes.

Em Silva et al. (2007), considera-se que o modelo de carregamento proposto por Murray, Allesn e Ungar (1997) é mais satisfatório para simular as forças dinâmicas de- 
Tabela 3 - Tipos de atividades e aplicabilidades em diversos tipos de estruturas.

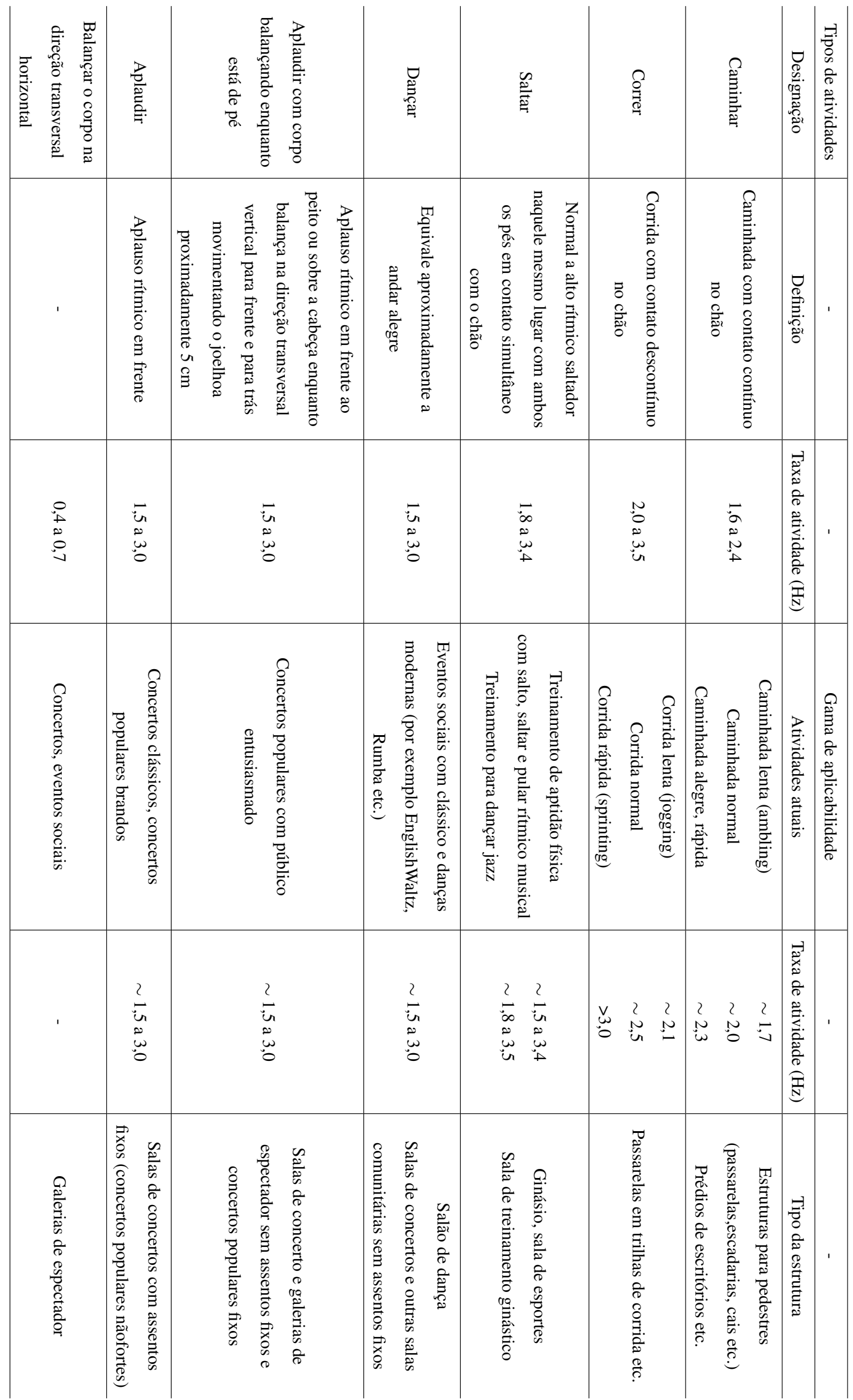

Fonte: Bachmann et al. (1995). 
vido aos pedestres caminhando, pois, as representações destas ações dinâmicas são mais realísticas, porém casos de carregamento que envolvem mais do que um pedestre caminhando nas passarelas devem ser investigados tomando como base esse modelo. Segundo Figueiredo et al. (2008), os modelos de carregamentos apresentados, que incorporam a variação espacial e temporal, são mais realísticos porque a ação dinâmica é mudada de acordo com a posição do indivíduo sobre a estrutura.

$\mathrm{Na}$ Tabela 3 tem-se um resumo que esclarece de forma abrangente a relação dos vários tipos de atividade com sua aplicabilidade em diversos tipos de estruturas.

\section{Considerações finais}

O estudo teórico abordado nesse trabalho foi realizado a partir de revisões bibliográficas e pode ser aplicado em diversos tipos de estruturas, pois incorpora os conceitos relacionados aos carregamentos de pedestres. Tal estudo foi fundamental porque permitiu um entendimento abrangente do comportamento das estruturas quando submetidas aos movimentos de caminhada, corrida, etc. dos pedestres sobre as mesmas.

A partir das propostas de modelagem abordadas nesse estudo é possível realizar a simulação numérica do movimento de caminhada em estruturas, sendo uma alternativa viável que possibilita identificar as ressonâncias referentes às frequências naturais nas passarelas de madeira, aço e concreto que ocorrem nas direções transversal vertical e transversal horizontal devido aos diversos tipos de caminhada. A simulação numérica possibilita avaliar os efeitos de ressonância dos harmônicos devido ao movimento dos diversos tipos de caminhada.

Os modelos apresentados para os diversos tipos de carregamentos de pedestres permitem trabalhar com até cinco harmônicos e caracterizam a ação dos pedestres ou apenas considera um harmônico levando em conta que seja o harmônico com maior efeito de ressonância, isto é, foi observado que as propostas de modelagem apresentadas diferenciam-se, principalmente, pelo número de harmônicos considerados na ação dos pedestres.

Constatou-se que a maioria das propostas de modelagem realizadas pelos diversos autores refere-se a componente da ação na direção transversal vertical e trata da caminhada com frequência na faixa de 1,6 a 2,4 Hz, enquanto que propostas de modelagem para componentes da ação nas direções transversal horizontal e longitudinal ainda são pouco estudadas.

\section{Referências}

AMERICAN ASSOCIATION OF SATATE HIGHWAY AND TRANSPORTATION OFFICIALS. AASHTO: guide specifications for design of pedestrian bridges. USA, aug. 1997.

ANDRIACCHI, T. P.; OGLE, J. A.; GALANTE, J. O. Walking speed as a basis for normal and abnormal gait measurements. Journal of Biomechanics, New York, v. 10, p. 261-268, 1977.

BACHMANN, H. "Lively" footbridges-a real challenge. In: INTERNATIONAL CONFERENCE ON DESIGN AND DYNAMIC BEHAVIOUR OF FOOTBRIDGES FOOTBRIDGE 2002, 1., 2002, Paris. Proceedings... Paris, France, nov. 2002.

BACHMANN, H.; AMMANN, W. Vibrations in structures: induced by man and machines. Zurich: IABSE, 1987.

BACHMANN, H.; PRETLOVE, A. J.; RAINER, H. Dynamic forces from rhythmical human body motions. In: BACHMANN, H.; AMMANN, W. J.; DEISCHL, F.; EISENMANN, J.; FLOEGL, I.; HIRSCH, G. H.; KLEIN, G. K.; LANDE, G. J.; MAHRENHOLTZ, O.; NATKE, H. G.; NUSSBAUMER, H.; PRETLOVE, A. J.; RAINER, J. H.; SAEMANN, E. U.; STEINBEISSER, L. Vibration problems in structures: practical guidelines. Basel: Birkhauser, 1995. (Appendix G).

BLANCHARD, J.; DAVIES, B. L.; SMITH, J. W. Design criteria and analysis for dynamic loading of footbridges. Report SR: Transport and Road Research Laboratory, Crowthorne, v. 275, 1977.

BRITISH STANDARDS. BS5400: steel, concrete and composite bridges: specification for loads. London, UK, 1978. Part 2, Appendix C.

BRO 2004. Publikation 2004:56: Vägverkets allmänna tekniska beskrivning för nybyggande och förbättring av broar. Svensk Byggtjänst, Stockholm, Sverige, 2004.

COMITÉ EURO-INTERNATIONAL DU BÉTON. CEBFIP. Bulletin D'Information, England, n. 209, Aug. 1993.

EBRAHIMPOUR, A.; HAMAM, A.; SACK, R. L.; PATTEN, W. N. Measuringand modelingdynamic loads imposed by movingcrowds. Journal of Structural Engineering, New York, v. 122, n. 12, p. 1468-1474, 1996.

EBRAHIMPOUR, A.; SACK, R. L.; PATTEN, W. N.; HAMAM, A. Experimental measurements of dynamic 
loads imposed by moving crowds. In: STRUCTURES CONGRESS, 12., ASCE, 1994. Proceedings... Atlanta, Georgia, USA, Apr. 1994.

EUROPEAN COMMITTEE FOR STANDARDIZATION. EUROCODE 5: design of timber structures. Part 2: Bridges. Brussels, Belgium, 1995.

EUROCODE 5: design of timber structures. Part 2: Bridges. London, UK, 2004.

EYRE, R.; CULLINGTON, D. W. Experience with vibration absorbers on footbridges. Research Report: Transport and Road Research Laboratory, Crowthrone, n. 18, 1985.

FIGUEIREDO, F. P. Estudo do comportamento dinâmico de passarelas devido ao caminhar de pedestres. 2005. 122 f. Dissertação (Mestrado em Engenharia Civil) - Universidade Estadual do Rio de Janeiro, Faculdade de Engenharia, Rio de Janeiro, 2005.

FIGUEIREDO, F. P.; SILVA, J. G. S.; VELLASCO, P. C. G. S.; LIMA, L. R. O.; ANDRADE, S. A. L. A parametric study of composite footbridges under pedestrian walking loads. Engineering Structures, Oxford, v. 30, p. 605-615, 2008

FUJINO, Y.; PACHECO, B. M.; NAKAMURA, S.; WARNITCHAI, P. Synchronization of human walking observed during transversal horizontal vibration of a congested pedestrian bridge. Earthquake Engineering and Structural Dynamics, Sussex, v. 22, n. 9, p. 741-758, 1993.

GALBRAITH, F. W.; BARTON, M. V. Ground loading from footsteps. Journal of Acoustical Society of America, Lousiana, v. 48, n. 5, p. 1288-1292, 1970.

INTERNATIONAL ORGANIZATION FOR STANDARDIZATION. ISO 10137: bases for design of structures - serviceability of buildings and pedestrian walkways against vibration. Geneva, Switzerland, 2005

KERR, S. C.; BISHOP, N. W. M. Human induced loadingon flexible staircases. Engineering Structures, Oxford, v. 23, p. 37-45, 2001.

MATSUMOTO, Y.; NISHIOKA, T.; SHIOJIRI, H.; MATSUZAKI, K. Dynamic design of footbridges. In: IABSE, 1978, Zurich, Switzerland. Proceedings. . Z Zurich, Switzerland, 1978. v. 2.

MATSUMOTO, Y.; SATO, S.; NISHIOKA, T.; SHIOJIRI, $\mathrm{H}$. A study on design of pedestrian over-bridges. Transactions of JSCE, Tokyo, v. 4, p. 50-51, 1972.
MURRAY, T. M.; ALLEN, D. E.; UNGAR, E. E. Floor vibrations due to human activity. Steel Design Guide Series, Chicago, n. 11, 1997.

NAKAMURA, S. I. Transversal horizontal vibration on a pedestrian cable-stayed bridge. Journal of Structural Engineering International, Zurich, v. 12, n. 4, p. 295-300, 2002.

ONTARIO HIGHWAY BRIDGE DESIGN CODE. OHBDC: ministry of transportation and communications highway engineering division. 3rd ed. Ontario, 1991.

RAINER, J. H.; PERNICA, G.; ALLEN, D. E. Dynamic loading and response of footbridges. Canadian Journal of Civil Engineering, Ottawa, v. 15, n. 1, p. 66-71, 1988.

SILVA, J. G. S.; VELLASCO, P. C. G. S.; ANDRADE, S. A. L.; LIMA, L. R. O.; FIGUEIREDO, F. P. Vibration analysis of footbridges due to transversal vertical human loads. Computers \& Structures, Elmsford, v. 85, p. 1693 1703, 2007.

SKORECKI, J. The design and construction of a new apparatus for measuring the transversal vertical forces exerted in walking: a gait machine. Journal of Strain Analysis, London, v. 1, n. 5, p. 429-438, 1966.

SMITH, J. W. Vibrations in structures: applications in civil engineering design. London, UK: Chapman and Hall, 1988.

SWISS NATIONAL STANDARDS. SIA 160: effects of loads on structures, 1989.

VARELA, W. D. Modelo teórico-experimental para análise de vibrações induzidas por pessoas caminhando sobre lajes de edifícios. 2004. 309 f. Tese (Doutorado em Engenharia Civil) - Universidade Federal do Rio de Janeiro, Rio de Janeiro, 2004.

WHEELER, J. E. Pedestrian-Induced vibrations in footbridges. In: AUSTRALIAN ROAD RESEARCH BOARD (ARRB) Conference, 10., 1980, Sydney, Australia. Proceedings... Sydney, Australia, Aug. 1980.

WHEELER, J. E. Prediction and control of pedestrianinduced vibration in footbridges. Journal of the Structural Division, New York, v. 108, n. 9, p. 2045-2065, 1982.

WRIGHT, D. T.; GREEN, R. Human sensitivity to vibration: report. Kingston: Queen’s University, 1959.

YAO, S.; WRIGHT, J.; PAVIC, A.; REYNOLDS, P. Forces generated when bouncing or jumping on a flexible Structure. In: INTERNATIONAL CONFERENCE ON NOISE 
AND VIBRATION ENGINEERING, 2002, Leuven, Belgium. Proceedings... Leuven, Belgium, Sept. 2002.

YOUNG, P. Improved floor vibration prediction methodologies. "Engineering for Structural Vibration - Current developments in research and practice”. In: ARUP VIBRATION SEMINAR, 2001. Proceedings. Institution of Mechanical Engineers, Oct. 2001. 